\title{
Memory for scripts in young and older adults
}

\author{
LEAH L. LIGHT \\ Pitzer College, Claremont, California \\ and \\ PATRICIA A. ANDERSON \\ Claremont Graduate School, Claremont, California
}

\begin{abstract}
This study examined the question of whether young and older adults differ in their representation or utilization of the generic knowledge contained in scripts. In Experiment 1, young and older adults generated scripts for routine daily activities, such as grocery shopping, going to the doctor, and writing a letter to a friend. No evidence was found for age-related differences in the way that stereotypical action sequences are represented in semantic memory. In Experiment 2, young adults were found to recall and recognize new instantiations of scripts better than did older adults. However, adults in both age groups displayed similar effects of action typicality on retention, suggesting that there are no age-related differences in drawing inferences from generic knowledge. The implications of these findings for processing-resource hypotheses about memory and aging are discussed.
\end{abstract}

There is considerable evidence that older adults do not remember sentences or prose as well as do young adults (Cohen, 1979, 1981; Dixon, Simon, Nowak, \& Hultsch, 1982; Feier \& Gerstman, 1980;Gordon \& Clark, 1974; Light, Zelinski, \& Moore, 1982; Simon, Dixon, Nowak, \& Hultsch, 1982; Taub, 1979; Taub \& Kline, 1978). Although the research has generally not been analytic as to the source of the observed age differences, there is some support for the view that reduced workingmemory capacity in older adults leads to difficulty in memory for discourse (e.g., Cohen, 1979, 1981; Light, Zelinski, \& Moore, 1982). One consequence of reduced working-memory capacity in older adults may be less efficient integration of new information with previously stored general world knowledge. People generally do not state explicitly information that they believe can be inferred readily by listeners or readers on the basis of general world knowledge (Clark \& Haviland, 1977; Grice, 1975; Harris \& Monaco, 1978; Minsky, 1975; Schank \& Abelson, 1977). Therefore, problems in using general world knowledge can lead to difficulties in comprehension and in subsequent retention of spoken or written discourse (e.g., Bransford \& Johnson, 1972).

There have been only a few investigations of older adults' ability to use general world knowledge in comprehension and memory for discourse. Unfortunately, these have produced ambiguous or conflicting results.

This research was supported by National Institute on Aging Grant 1 R01 AG02452. We are indebted to Deborah M. Burke for insightful comments on an earlier version of this paper, as well as for numerous discussions of this work. Requests for reprints should be addressed to Leah L. Light, Department of Psychology, Pitzer College, Claremont, California 91711.
For instance, Till and Walsh (1980) reported that older adults do not spontaneously encode the pragmatic implications of sentences. In their studies, people heard sentences such as "The chauffeur drove on the left side" and were later tested either by free recall or by recall cued with a noun referring to information that could be inferred from a sentence (e.g., "England"). In two studies, there was an interaction between age and recall condition, such that older adults performed less well on cued recall than on free recall, though this was not true for young subjects. However, in a third experiment, the cues were equally effective for both age groups when subjects were required to give, immediately after a sentence had been presented, a one-word response that reflected sentence meaning. This suggests that older adults can use prior world knowledge effectively under at least some conditions. In the only study to examine on-line semantic processing of pragmatic implications (Burke \& Yee, in press), sentences were presented visually one word at a time, and subjects were required to perform a lexical-decision task after each sentence. When the lexical-decision target was an instrument implied by the sentence, latencies were faster for both older and young adults than when the target was an unrelated word, suggesting that older adults process semantic implications as well as young adults do.

Belmore (1981) and Cohen (1981, Experiment 2) compared the ability of young and older adults to answer questions about passages in which information was either stated explicitly or had to be inferred from general world knowledge. Cohen reported that the two age groups did not differ in memory for explicit facts but that older adults made more errors on items requiring inferences. On the other hand, Belmore found age 
differences on a delayed test for both explicitly stated and implied information but no age differences when people were tested immediately after having read passages. Belmore's results, taken together with those of Burke and Yee (in press), suggest that older adults do not have problems in accessing real-world knowledge during comprehension but rather that the problem lies in a more general memory impairment. This would explain Cohen's (1979, Experiment 2) finding that older adults were less accurate than young adults in detecting when short passages contained statements that contradicted common knowledge about the world (e.g., that a blind man read a newspaper or that a housewife who had no bread made sandwiches). In this study, there was no measure of memory for information in the passages; it is therefore possible that failure to detect anomaly resulted from problems in memory for explicit information rather than from failure to access or utilize pragmatic information in long-term memory.

In this paper, we report an experiment that bears on the question of whether young and older adults differ in the extent to which they utilize real-world knowledge in recall and recognition of discourse. Young and older adults were exposed to a story about several days in the life of a character named Jack, who engages in a set of mundane activities such as writing a letter to a friend and going grocery shopping. Schank and Abelson (1977) suggested that we represent our knowledge of such stereotypic action sequences as "scripts." These scripts specify conventional roles, props, action sequences, reasons for engaging in an activity, and expected outcomes for ordinary activities. For example, we expect to find waiters and menus in restaurants, we go to restaurants because we are hungry, we eat, and then we leave after having paid the bill. There is evidence that scripts play an important role in comprehension and memory for particular instances of conventional activities. Understanding requires people to match what they see or hear to a previously stored representation of actions. Thus, new information is understood in terms of old information. Details that are omitted from a particular instantiation of a script are inferred on the basis of the stereotypic sequence that defines well-known situations. For this reason, college students are likely to infer that highly typical actions have taken place even when these have not been explicitly stated and to later intrude these typical actions in recall and to make false alarms to them in recognition (e.g., Bower, Black, \& Turner, 1979; Graesser, Gordon, \& Sawyer, 1979; Graesser, Woll, Kowalski, \& Smith, 1980). Such findings can be explained in terms of the "script pointer plus tag" hypothesis of Schank and Abelson (1977) or the partial copy model of Bower et al. (1979). These hypotheses have in common the notion that when an instantiation of a particular activity is encountered, its generic script is activated in semantic memory. In addition, the specific activities mentioned (Bower et al., 1979), or at least those that deviate from the generic scripts (Schank \& Abelson, 1977), are recorded in episodic memory. Highly typical actions are likely to be recalled or (falsely) recognized even when they have not been stated explicitly, because they are activated as part of the generic script. Hence, when scripts are invoked, typical actions are discriminated less well than are atypical ones.

Comparing the performance of young and older adults on memory for scripts provides a testing ground for hypotheses about why older adults remember discourse less well than do young adults. Here we will consider two hypotheses about age-related differences in memory for scripts. These hypotheses share the premise that older adults have reduced processing resources; they differ in the hypothesized consequences of reduced attentional capacity.

The first hypothesis we examine is that of Cohen (1979), who argued that, due to reduced capacity in working memory, older adults have problems in integrating new information with prior knowledge. This hypothesis is related to the more general claim that older adults have a deficit in semantic processing (e.g., Craik \& Simon, 1980; Eysenck, 1974; Simon, 1979) that results in poorer comprehension and memory. If older adults are less likely to access general world knowledge, that is, generic scripts, during comprehension of discourse, they should be less likely than young adults to infer falsely that highly typical actions take place when these are not stated explicitly. As a result, older adults should exhibit smaller typicality effects than those that young adults exhibit and should be more accurate than are young adults in discriminating typical actions that have been mentioned from those that have not been mentioned.

A second, and rather different, set of predictions is derived from the work of Rabinowitz, Craik, and Ackerman (1982). These authors proposed that reduced processing resources in the elderly result in decreased likelihood that the specific meanings of events in context are encoded, because encoding information in context is effortful, whereas encoding of generic meaning is relatively automatic (Hasher \& Zacks, 1979). Although Rabinowitz et al. dealt with memory for single words, their hypothesis has implications for script memory as well. Saying that the general meaning of a script is encoded is theoretically equivalent to saying that the generic script has been activated in semantic memory. Similarly, failure to encode the meaning of a word in context can be viewed as being equivalent to failure to record the details of the instantiation of a script on a particular occasion. Because of reduced capacity, then, older adults would be expected to encode all instantiations of a script in more or less the same stereotyped way and to be less likely to encode each instantiation in a distinctive, contextually specific way. Thus, the recall protocols of older adults should contain principally typical script information (both 
explicitly stated and inferred). Also, the recognition performance of older adults should be poorer for both typical and atypical script actions. That is, older adults would be expected to make more hits and more false alarms on typical script actions than younger adults (because of confusion as to which actions occurred) but fewer hits on atypical actions (because these actions would not have been encoded as accurately in episodic memory). Neither age group would be expected to make many false alarms on atypical actions.

The predictions outlined above are reasonable only if young and older adults represent scripts the same way in semantic memory, that is, if actions deemed to be typical by one age group are also viewed as being typical by the other. For this reason, in Experiment 1 we gathered script norms from young and older adults for use in constructing materials for the script-memory study reported as Experiment 2.

\section{EXPERIMENT 1}

The main purpose of Experiment 1 was to gather normative data from young and older adults so that suitable materials could be prepared for Experiment 2. There were two phases to the research. In the first phase, we asked samples of young and older adults to generate scripts for specified situations. In the second phase, independent samples of young and older adults rated a subset of the items generated for each script on typicality, that is, the likelihood that the actions mentioned would occur in an instantiation of the script.

In addition to providing materials for use in Experiment 2 , the results of this study should be interesting in their own right. There have been no studies comparing the structure of information in semantic memory in young and older adults that have dealt with stimuli more complex than single words or word triads in wordassociation tasks (e.g., Howard, 1980; Lovelace \& Cooley, 1982; Perlmutter, 1978; Riegel \& Birren, 1966; Riegel \& Riegel, 1964). Some investigators have reported greater variability of responses by older adults on word-association tasks (e.g., Lovelace \& Cooley, 1982; Perlmutter, 1979; Riegel \& Riege1, 1964), whereas others have not observed this result (e.g., Howard, 1980, Note 1; Perlmutter \& Mitchell, 1982; Burke \& Peters, Note 2). Also, older adults have generally been found to be less fluent, that is, to produce fewer responses in timed word-association tasks (e.g., Birren, Riegel, \& Robbin, 1962; Howard, 1980; Riegel \& Birren, 1966; Schaie \& Parham, 1977; Schaie \& Strother, 1968; Schonfield \& Stones, 1979; Stones, 1978; but see Drachman \& Leavitt, 1972, for a different conclusion), although Perlmutter (1978) found no effects of age on the number of associates produced when subjects worked at their own pace. The script-generation data collected in the first phase of Experiment 1 will permit us to determine whether older adults exhibit more variability in their representation of common activities and whether young and older adults differ in the number of actions included in their script productions.

\section{Method}

Subjects. In the first phase of this study, 46 young adults ( 33 females and 13 males) and 56 older adults ( 36 females and 20 males) generated scripts. The young subjects (mean age $=$ 27.00 years, range $=19-38$ years) were students attending colleges in southern California, and the older subjects (mean age $=68.68$ years, range $=61-81$ years) were participating in an Elderhostel program at Pitzer College. The two groups were equivalent with regard to education. The young subjects had had a mean of 16.48 years of schooling, and the older adults had had a mean of 16.06 years. The 40 -item vocabulary subtest from the Shipley Hartford Institute of Living Scale (Shipley, 1940) was administered to assess verbal ability. As is typically found, the older group scored reliably higher than the young group, with means of 36.91 and 33.15 for the older and the young adults $[\mathrm{t}(100)=5.44, \mathrm{p}<.05]$. In the typicality rating phase of the study, there were 19 young adults (13 females and 6 males) and 20 older adults ( 12 females and 8 males). The young subjects (mean age $=26.67$ years, range $=22-31$ years) were primarily undergraduate and graduate students at the Claremont Colleges, and the older adults (mean age $=68.70$ years, range $=$ 57-78 years) were mostly Pomona College alumni. The two groups were well-matched in education, with the young group having had a mean of 16.78 years of schooling and the older group having had a mean of 17.63 years. Again, the older adults . $($ mean $=38.15)$ achieved reliably higher scores on the ShipleyHartford vocabulary scale than the young adults (mean $=36.50$ ) $[\mathrm{t}(34)=2.17] .^{1}$

Materials and Procedure. In the generation phase of the experiment, normative data were gathered on six routine activities: writing a letter to a friend, going to the doctor, grocery shopping, getting up in the morning, visiting a friend in the hospital, and going to a wedding. Each subject contributed three scripts, with approximately half of the subjects in each age group writing on the first three mentioned activities and the remaining subjects writing on the other three.

Each subject received a test booklet. The cover sheet gave general instructions based on those used by Bower, Black, and Turner (1979). These instructions emphasized that the task was to produce a list of common actions or events for each of three activities and that subjects should not include idiosyncratic actions based on their own behavior but should list actions that would be typically performed by most people. A detailed example of the typical actions involved in going to a class followed on the second page. (This example was salient for both age groups, since the young adults were enrolled in college and the older adults were taking minicourses in the Elderhostel program.) Each of the three remaining pages of the booklet gave instructions for a particular activity. For instance, subjects might be asked to "write a list of actions describing what people generally do when they go grocery shopping," beginning with "enter the store" and ending with "leave the store." Opening and closing actions were provided for each activity to indicate the time frame for the script. Each page contained 23 additional numbered lines, and the subjects were asked to produce about 20 actions for each activity. Three random orders of each of the sets of the three activities were used approximately equally often.

The subjects worked on the task at their own rate, taking from 20 to $50 \mathrm{~min}$ to complete three scripts. They were tested in groups of 5 to 15 individuals of the same age group.

From the items generated by the subjects in the first phase of the research, we selected actions to be rated for typicality. Actions were chosen in such a way that the full range of generation frequencies (see below) would be represented. Items that 
duplicated the semantic content of other items (e.g., took a bath, took a shower) or were inconsistent with items from the same script were excluded. In addition to items generated by the subjects, some actions that no subject had produced but that we judged to be possible (although not typical) within the context of a scripted activity (e.g., "moaned" for "getting up in the morning") were included. For each scripted activity, actions were listed in a naturalistic sequence to tell a (very boring) story. The number of actions to be rated varied from 53 to 62 per script.

The subjects were tested individually or in pairs. Each subject rated three scripts, with about half the people in each age group rating items from "writing a letter," "going to the hospital," and "grocery shopping," and the remaining people rating items from "going to the doctor," "getting up in the morning," and "going to a wedding." From 9 to 11 people in each age group rated each script. The instructions, based on those used by Graesser et al. (1980), requested the subjects to make a judgment as to how "typical or necessary" each item was for completion of an activity. A 6-point scale was used, with a rating of 1 indicating a "very atypical" item and a rating of 6 indicating a "very typical" item.

\section{Results}

Script generation. It was necessary to discard the responses of two young and six old adults for one or two activities because they had failed to construe the script name correctly. For instance, two subjects, one young and one old, described a visit to the doctor from the point of view of a doctor rather than of a patient. Also, five people, one young and four old, described the events that might take place at a wedding reception rather than at a wedding ceremony. We could discern no qualitative differences in the ways that people of different ages misconstrued the task. The number of people in each age group who contributed data to the analysis is given in Table 1 for each script.

The first step in the analysis was to count, for each script, the number of times each action was mentioned. Separate counts were made for young and older adults. Actions generated by different subjects were treated as tokens of the same type if they conveyed the same meaning (e.g., "say hello to the receptionist" and "greet the receptionist"). Two measures were then computed for each subject: (1) the number of actions produced for each script and (2) the proportion of the subject's responses for that script that were unique (produced by only that one subject). For each script, the mean number of items generated and the mean proportion of unique items are presented as a function of age in Table 1 . There were no reliable age differences by $t$ test on either measure for any of the six scripts (all ps $>.05$ ). Thus, under untimed conditions, young and older adults did not differ in either the amount of detail with which they described common activities or in the variability of their representations of these events.

A more detailed analysis of the items generated for each script was also performed. The proportions of young and older adults producing a particular action were calculated. The correlations between these proportions for each of the six scripted activities are given in Table 1. These correlations ranged from .72 to .87 and were all highly reliable.

Typicality ratings. Mean typicality ratings were calculated for each item for each script for young and older subjects separately and for the two age groups combined. The typicality ratings of young and older adults were very similar; the correlations between the two sets of typicality ratings ranged from .83 to .89 for the six scripts (see Table 2). These correlations are impressive in light of the small number of people in each age group who rated a given script. In addition to computing these correlations, we also compared the mean typicality ratings of young and older adults for each action in every script. Over the 346 comparisons, we found 36 differences $(10 \%)$ reliable at the .05 level. Closer scrutiny of the items for which the means differed did not reveal any obvious pattern of age differences in typicality judgments.

We might expect that people will generate highly typical actions more often than they will atypical actions. For the six activities we examined, the correlations between generation frequency and typicality were high both within each age group and for the combined age groups (see Table 2) and were also similar across ages. The correlations ranged from .46 to .66 for the young group and from .41 to .64 for the older group. With the two age groups combined, the correlations ranged from .51 to .68 . These correlations are all considerably higher than the .10 reported by Graesser et al. (1980). However, the range of typicality in Graesser et al.'s (1980) sample of actions was very narrow, whereas our sample included actions with mean typicality ratings from 1.1 to 6.0 .

Table 1

Script Generation

\begin{tabular}{|c|c|c|c|c|c|c|c|}
\hline \multirow[b]{2}{*}{ Script Name } & \multicolumn{2}{|c|}{ No. of Subjects } & \multicolumn{2}{|c|}{$\begin{array}{c}\text { Mean No. of Items } \\
\text { Generated }\end{array}$} & \multicolumn{2}{|c|}{$\begin{array}{c}\text { Mean Proportion } \\
\text { of Unique Items } \\
\end{array}$} & \multirow[b]{2}{*}{ Correlation* } \\
\hline & Young & Old & Young & Old & Young & Old & \\
\hline Writing a Letter & 22 & 27 & 16.36 & 17.04 & .21 & .19 & .85 \\
\hline Going to the Doctor & 21 & 26 & 17.86 & 18.12 & .23 & .22 & .85 \\
\hline Going Grocery Shopping & 22 & 27 & 18.27 & 20.40 & .26 & .26 & .76 \\
\hline Getting up in the Morning & 24 & 29 & 20.54 & 20.41 & .16 & .17 & .83 \\
\hline Going to the Hospital & 24 & 27 & 15.58 & 16.52 & .19 & .17 & .87 \\
\hline Going to a Wedding & 23 & 25 & 15.17 & 16.32 & .26 & .18 & .72 \\
\hline
\end{tabular}

*Correlation between generation frequencies of young and old groups. 
Table 2

Typicality Judgment Correlations

\begin{tabular}{lccccc}
\hline \multicolumn{1}{c}{ Script Name } & $\begin{array}{c}\text { Correlation of Mean Typicality } \\
\text { Ratings for Young and Old }\end{array}$ & \multicolumn{3}{c}{$\begin{array}{c}\text { Correlation of Typicality Ratings with } \\
\text { Generation Frequency }\end{array}$} \\
\cline { 4 - 6 } & .89 & .66 & Old Only & Combined \\
\hline Writing a Letter & .84 & .57 & .64 & .68 \\
Going to the Doctor & .83 & .52 & .41 & .52 \\
Going Grocery Shopping & .88 & .58 & .47 & .51 \\
Getting up in the Morning & .87 & .57 & .53 & .54 \\
Going to the Hospital & .85 & .46 & .58 & .58 \\
Going to a Wedding & & & & .58 \\
\hline
\end{tabular}

\section{Discussion}

The results of this study are straightforward. We found no evidence of age-related differences in the mental representation of conventional activities. First, young and older adults produced about the same number of actions when asked to describe scripted activities. It could be argued that this is simply an artifact of our instructions, which asked people to list about 20 actions per script. However, the mean number of actions generated varied considerably from activity to activity and was generally lower than 20 . Thus, under untimed conditions, the amount of detail with which activities are described does not seem to be related to age. Second, there were no reliable age differences in the proportion of unique actions that people produced in describing everyday activities. This result joins an increasing body of literature suggesting that young and older adults are similar in between-subject variability in responses on a variety of verbal production tasks (Howard, 1980, Note 1; Perlmutter \& Mitchell, 1982; Burke \& Peters, Note 2). Third, young and older adults were in very close agreement in their ratings of individual actions for typicality. Finally, the correlations between rated typicality and generation frequency were similar for the two age groups. Thus, we can be confident that any interactions between age and typicality that we may find in the memory tasks used in Experiment 2 are not the result of systematic age differences in the way that scripts are represented in semantic memory.

\section{EXPERIMENT 2}

In Experiment 2, we compared the recall and recognition of young and older adults for scripted activities. As discussed in the introduction, hypotheses that older adults suffer from impaired semantic processing that prevents efficient integration of general world knowledge with new information during comprehension and subsequent memory of discourse predict that older adults will demonstrate reduced effects of typicality in recall and recognition of conventional activities. On the other hand, hypotheses that older adults access realworld knowledge as well as young adults do but have reduced ability to encode specific instantiations of scripts predict that older adults will exhibit greater dependence on general knowledge and will therefore remember less typical actions less well. Thus, these two classes of hypotheses both predict interactions of age with typicality, but the predicted interactions are of different forms.

\section{Method}

Subjects. Twenty-four young adults (13 females and 11 males) and 24 older adults (12 females and 12 males) participated in this experiment. The young adults (mean age $=$ 24.58 years, range $=20-33$ years) were alumni, graduate students, and advanced undergraduates of the Claremont Colleges, and the older adults (mean age $=72.63$ years, range $=61-84$ years) were alumni of Pomona College. Both groups were highly educated; the young subjects had had a mean of 16.79 years of schooling, and the older subjects had had a mean of 17.38 years. As we found before, the older group performed reliably better than the young group on the Shipley-Hartford vocabulary subtest, with means of 37.29 and 34.21 for the two groups, respectively $[\mathrm{t}(46)=3.33]$.

Materials. Following the procedure of Graesser et al. (1980), we constructed a story about a character named Jack. The story consisted of a sequence of six scripted activities. There were four experimental scripts, with one additional buffer script at the beginning of the story and one at the end. The experimental scripts are summarized by the following titles: getting up in the morning, going to the doctor, writing a letter to a friend, and going to a hospital to visit a friend. In the Jack story, each script was identified by a sentence that contained the script title (for example, Jack decided to visit his friend in the hospital) before the actions for that script were listed. The four experimental scripts averaged 105 words in length.

Two versions of the Jack story, each having different samples of items for the four experimental scripts, were produced for purposes of counterbalancing. Each experimental script in each version contained 12 test items and a variable number of filler actions needed for continuity. The 12 experimental actions were drawn from the items rated for typicality in Experiment 1. From three to five actions per script fell into each of three categories of typicality: typical (mean rating $=5.5$, range $=4.9$ to 6.0 ), average (mean rating $=3.9$, range $=2.9$ to 4.7 ), and atypical (mean rating $=1.9$, range $=1.1$ to 2.8 ). The typicality means were based on the combined ratings of the young and older adults in Experiment 1, because the ratings of the two age groups were very similar.

The two versions of the Jack story were recorded at a rate of 130 words per minute.

Procedure. The subjects were tested alone or in groups of two or three people of the same age group. They were asked to "listen to a story about a character named Jack and the activi- 
ties he performed over a series of several days" and to follow along with a written version of the story. They were instructed to listen closely to the story because they would be asked questions about Jack's personality, as well as about the story itself. Immediately after presentation of the story, the subjects were given a $10-15$-min filler task consisting of a 10-item multiplechoice questionnaire about various aspects of Jack's personality After the filler task, the subjects were first tested for recall of two of the four experimental scripts and were then tested for recognition memory for the remaining two experimental scripts.

For recall, the subjects received a test booklet consisting of two pages, one for each script tested. Each page gave the title of the script and a short description of the activity (e.g., "one of the things Jack did was visit his friend in the hospital"). The written instructions asked the subjects to list as many things as they could remember that Jack did or that happened to Jack while he performed a particular activity; the instructions stressed that the subjects were to list only events that were explicitly mentioned in the story. Beginning and ending actions (e.g., "Jack arrived at the hospital" and "Jack left the hospital") were provided.

The recognition test booklet also consisted of two pages, one per tested script. Each page gave the title and a short description of the script and listed the 24 test items from the script (12 from each of the two versions) in random order. The written instructions made it clear that some of the items had been explicitly mentioned in the Jack story and that others had not. The subjects rated their confidence that each item had been presented on a 6-point scale, with 1 representing "very sure the item was presented" and 6 representing "very sure the item was not presented."

The version of the Jack story and the particular scripts chosen for recall and recognition were counterbalanced for each age group. The subjects were given $3 \mathrm{~min}$ for recall or recognition of each script. This amount of time was more than adequate for all individuals tested.

\section{Results}

Recall. For each subject, we computed the proportions of correctly recalled typical, average, and atypical actions. Mean proportions are given as a function of age and typicality in Table 3 . Inspection of Table 3 reveals that young adults $(.46)$ recalled a higher proportion of items correctly than did older adults (.37) and that typical items were recalled less often than average or atypical ones. These visual impressions were confirmed by a 2 (ages) x 3 (typicality) analysis of variance that yielded reliable main effects of age $[\mathrm{F}(1,46)=4.41$, $\mathrm{MSe}=.059]$ and typicality $[\mathrm{F}(2,92)=11.90$, MSe $=$ .033]. There was, however, little evidence of any interaction between age and typicality $(F=1.80)$.

Summary statistics for intrusion errors are given in
Table 3. For each subject, we computed the number of intrusions for the two scripts recalled by that subject. Although older adults made slightly more intrusion errors than did young adults, the difference was not reliable $[t(46)<1]$. For 29 of the 41 intrusions produced by young adults and 30 of the 53 intrusions produced by older adults, data on generation frequency and typicality were available from Experiment 1. Table 3 gives the mean proportion of individuals in Experiment 1 who produced one of these intrusions during script generation, as well as the mean typicality value of the intrusions separately for older and young adults. (These means are based on the combined data of young and older subjects in Experiment 1 for both generation probability and typicality.) Two things are immediately obvious in examining these means. First, intrusion errors tend to be highly typical actions that are also produced very often when subjects are asked to generate scripts. Second, there are clearly no age-related differences in either the number or nature of intrusion errors in recall.

Recognition. Confidence judgments of 1,2 , or 3 were counted as hits for those actions that appeared in the version of the Jack story to which a subject had been exposed and as false alarms for those actions that appeared in the other version. Mean proportions of hits and false alarms, as well as mean $\mathrm{d}^{\prime} \mathrm{s}{ }^{2}$ are given in Table 4 as a function of age and typicality. Separate analyses of variance were carried out on each of these three measures.

Although there was no main effect of age on falsealarm rate $[F(1,46)<1]$, there was a reliable age effect in the analysis of hit rates $[F(1,46)=4.95$, MSe $=.026]$, with young adults $(.83)$ achieving a higher hit rate than older adults (.77). The age difference was also reliable in the $\mathrm{d}^{\prime}$ analysis $[\mathrm{F}(1,46)=5.92$, MSe $=$ 1.01]. Thus, young and older adults appear to differ in the accuracy of their memory for routine stereotypical activities, with the effect due primarily to differences in hit rates.

The pattern of effects for typicality mirrors that found elsewhere for recognition (e.g., Graesser et al., 1979; Graesser et al., 1980). ${ }^{3}$ There was no effect of typicality on hit rate $[\mathrm{F}(2,92)=1.81, \mathrm{MSe}=.028]$, but there was a sizable effect of typicality on falsealarm rate $[\mathrm{F}(2,92)=92.37, \mathrm{MSe}=.027]$ (with less

Table 3

Script Recall

\begin{tabular}{|c|c|c|c|c|c|c|c|}
\hline & \multicolumn{4}{|c|}{ P(Recall) } & \multicolumn{3}{|c|}{ Intrusions } \\
\hline & Typical & Average & Atypical & Mean & $\begin{array}{c}\text { Mean No. } \\
\text { of Intrusions* }\end{array}$ & Mean GP & $\begin{array}{c}\text { Mean } \\
\text { Typicality }\end{array}$ \\
\hline $\begin{array}{l}\text { Young } \\
\text { Old }\end{array}$ & $\begin{array}{l}.33 \\
.29\end{array}$ & $\begin{array}{l}.55 \\
.39\end{array}$ & $\begin{array}{l}.49 \\
.44\end{array}$ & $\begin{array}{l}.46 \\
.37\end{array}$ & $\begin{array}{l}1.71(1.21) \\
2.21(1.25)\end{array}$ & $\begin{array}{l}.58 \\
.57\end{array}$ & $\begin{array}{l}5.43 \\
5.40\end{array}$ \\
\hline Mean & .31 & .47 & .46 & .41 & $1.96(1.23)$ & .57 & 5.41 \\
\hline
\end{tabular}

Note-GP = proportion of subjects generating the intrusion as a response in Experiment $1 . \quad$ *The figures in parentheses are the mean numbers of items generated as intrusions for which typicality ratings were available from Experiment 1 . 
Table 4

Script Recognition

\begin{tabular}{|c|c|c|c|c|c|c|c|c|c|}
\hline & \multicolumn{3}{|c|}{$\mathrm{P}$ (Hit) } & \multicolumn{3}{|c|}{ P(False Alarm) } & \multicolumn{3}{|c|}{$\mathrm{d}^{\prime}$} \\
\hline & Typical & Average & Atypical & Typical & Average & Atypical & Typical & Average & Atypical \\
\hline Young & .86 & .84 & .78 & .47 & .14 & .08 & 1.40 & 2.61 & 2.74 \\
\hline Old & .79 & .77 & .75 & .54 & .16 & .07 & .95 & 2.11 & 2.48 \\
\hline Mean & .83 & .80 & .76 & .51 & .15 & .08 & 1.17 & 2.36 & 2.61 \\
\hline
\end{tabular}

typical items receiving fewer false alarms) and on d' $[\mathrm{F}(2,92)=34.30, \mathrm{MSe}=.821]$. Given that recognition memory as measured by d' scores was worst for typical items, it is important to note that the performance of both young and older groups was better than chance even for this category of actions, with $t(23)=7.79$ for the young subjects and $t(23)=8.04$ for the older subjects. There were no interactions of age with typicality in any of the three analyses (all Fs $<1$ ).

\section{Discussion}

The overall pattern of results that we obtained in recall and recognition replicate the patterns obtained by other researchers. Recall of explicitly stated script actions was best for atypical items, and actions intruded in recall were highly typical (Bower et al., 1979; Graesser et al., 1980; Hastie \& Kumar, 1979). Similarly, less typical items were better discriminated in recognition memory, and the typicality effect was carried mainly in the false-alarm rate, with more typical items producing more false alarms (Bower et al., 1979; Graesser et al., 1979; Graesser et al., 1980). This configuration of results is consistent with the view that new information is interpreted in terms of prior knowledge and that details that are omitted from a particular instantiation of some script are inferred on the basis of the stereotypic qualities of these well-known activities.

Our finding of age-related differences in memory is also consistent with what previous research has found. Older adults had poorer memory for scripted activities than did young adults, as demonstrated by older adults' lower recall of explicitly stated script actions and by their lower hit rates and $\mathrm{d}^{\prime}$ values in recognition. However, there were no indications of any interaction of age with typicality of script actions in either recall or recognition. Both young and older adults recalled more atypical than typical actions and were more accurate in their discrimination of explicitly stated from unstated atypical actions. Moreover, the quantity and quality of their errors were highly similar. Young and older subjects did not differ in either the number of intrusions that they produced in recall or in their rates of making false alarms in recognition. Members of both age groups gave intrusions that were highly typical and likely to be generated by subjects asked to describe stereotypical routine activities. Also, the false alarms of young and older subjects were far more numerous for typical than for average or atypical items. Thus, there is evidence here of age-related differences in memory but no evidence for age-related differences in typicality effects, which would suggest differences in the extent to which general world knowledge is integrated with new information.

\section{GENERAL DISCUSSION}

The results of the two experiments reported here offer no support for hypotheses that young and older adults differ either in the way in which conventional activities are represented in semantic memory or in the extent to which activation of scripted information is involved in retention. In Experiment 1, we found that young and older adults asked to describe stereotypical activities were very similar in the number of actions that they listed, in the proportion of unique responses that they produced, and in the typicality ratings that they assigned to script actions. Moreover, the pattern of correlations between typicality and generation frequency was very similar across ages.

Hypotheses that older adults cannot or do not rely on general world knowledge during comprehension and memory of discourse predict that young and older adults should have different patterns of typicality effects, with older adults displaying less influence of typicality in their recall intrusions and less evidence of typicality effects in recognition. However, in Experiment 2, although overall performance was lower for older adults, we could find no hint of age-related differences in typicality effects on any measure that we could devise for recall or recognition. Both age groups were more accurate in recall of unusual script actions and in discriminating explicitly stated from unstated atypical script actions in recognition. Members of both age groups produced recall intrusions that were very typical and apt to be listed in script generation, and both age groups made more false alarms on typical items. In short, we found no reason to believe that age-related problems in recall or recognition are due to differences in the extent to which young and older adults integrate generic knowledge with new information.

Our results are also difficult to reconcile with hypotheses that older adults rely on generic knowledge of everyday activities to the extent that they simply respond to the instantiation of a script by activation of the generic script in semantic memory, with little or no 
memory for the particular actions described. This hypothesis predicts that older adults should show greater effects of typicality than young adults. Their recall protocols would be expected to contain primarily typical actions, both explicitly stated and inferred. Moreover, older adults would be expected to have little discrimination of explicitly stated and unstated typical actions in recognition, because both types of actions have been activated as part of a generic script. Also, they should not have much recognition memory for explicitly stated actions (as indexed by hit rate) that are atypical, but possible, within a script, because these have not been encoded as effectively. However, none of these outcomes was obtained. In recall, both young and older adults recalled more atypical than typical actions. In recognition, both age groups were less likely to respond with false alarms to atypical actions, and neither age group showed any effect of typicality on hits. There is, therefore, no evidence for this position.

The results of Experiment 2 might be interpreted as supporting the view that the use of generic information involves automatic processes that do not require attentional capacity, whereas encoding any type of specific information (regardless of typicality) is effortful and therefore taxes the reduced attentional capacity of older adults (Hasher \& Zacks, 1979). Such a hypothesis might be able to explain why we observed age-related differences in recall and recognition but no sign of differential use of scripts by young and older adults. However, we believe that it is necessary to assume not only that older adults have more limited attentional capacity than do young adults, but also that processes involved in comprehension and encoding compete for these resources. Cohen (1979) has articulated a similar position with respect to language comprehension; she argued that older adults have difficulty in simultaneously maintaining surface information from one part of a message while carrying out integrative operations on other parts. Here we are suggesting that processes involved in maintaining activation of pragmatic knowledge, such as is embodied in scripts, must compete for attentional capacity with processes involved in encoding information for subsequent retention.

The assumption that maintaining prior world knowledge in an activated state during language comprehension requires the allocation of attentional capacity receives support from recent work of Britton and Tesser (1982). In a series of experiments, Britton and Tesser found that people who have more knowledge in some domain are slower to perform a secondary task while engaged in mental operations within the domain of expertise than those who are less knowledgeable in that domain. Britton and Tesser suggested that experts need more attentional capacity to maintain their greater knowledge in an active state and that they will therefore have less capacity left over to use for other mental operations if the prior knowledge is in an active state. The implication of this finding is that maintaining any general world knowledge in an active state requires capacity. To the extent that older adults have reduced capacity, maintaining script information in an activated state will require allocation of a relatively greater amount of capacity than is required in young adults, leaving older adults with less capacity for encoding new information of any sort for subsequent retention. Our finding that young and older adults rely on generic information to about the same extent further suggests that operations involved in on-line discourse comprehension are given higher priority than are operations needed for encoding by whatever mechanisms are responsible for allocation of attention among possible activities. This seems reasonable given that the need to understand and make oneself understood during conversational exchanges probably does not change much across the adult years.

In addition to having less capacity to allocate, it is possible that older adults have a second handicap. In Experiment 2, the scripted activities people were asked to remember represent a domain that older adults, by virtue of having lived longer than young adults, have had more opportunities to experience. Older adults might be viewed as experts in the art of everyday living. When asked to remember a new instantiation of a routine activity, such as going grocery shopping, older adults might require more attentional capacity than young adults to maintain the appropriate script in the state of activation needed for successful language understanding. Thus, older adults not only might have less capacity to allocate, but also their richer representation of world knowledge may place a greater burden on the capacity they do have. We are inclined not to take this second hypothesis, which is based on a suggestion by Britton and Tesser, too seriously, however, because we found no evidence in Experiment 1 of any differences in the mental representations of the scripts of young and older adults.

There is one other issue that we need to address at this time. We have argued that young and older adults do not differ in their use of generic knowledge to make inferences when they encounter instantiations of scripts. These findings are consonant with those of other workers. For instance, Denney and Palmer (1981) examined the responses of young and older adults to questions about situations that might arise in everyday life, such as finding the interior of the refrigerator warm to the touch or receiving a threatening phone call. Although they found a curvilinear relationship between age and quality of response, with adults in their forties and fifties giving somewhat better solutions than those who were younger (twenties and thirties) or older (sixties and seventies), the magnitude of the differences observed was extremely small. In a similar vein, Charness (1981) reported that the ability to choose a move or to evaluate end-game positions varied with skill but not 
with age in chess players ranging in age from 16 to 64 years. Nevertheless, there is ample evidence that older adults do not perform as well as young adults when asked to draw inferences from new information (e.g., Cohen, 1979, 1981; Light et al., 1982). We believe that it is important to distinguish between inferences based on general world knowledge and inferences that require integration of new facts with little reference to generic knowledge. The available evidence suggests that older adults will have difficulty in reasoning primarily when they must both store and manipulate new information, but that reasoning in situations that permit inferences based on generic knowledge remains relatively constant across the adult years.

\section{REFERENCE NOTES}

1. Howard, D. V. Restricted word association norms for adults between the ages of 20 and 80 (Tech. Rep. NIA-79-1). Washington, D.C: Georgetown University, Cognition Laboratory, January 1979.

2. Burke, D. M., \& Peters, L. J. Word associations in young and old adults. Unpublished manuscript, 1982. (Available from D. M. Burke, Department of Psychology, Pomona College, Claremont, California 91711.

\section{REFERENCES}

Belmore, S. M. Age-related changes in processing explicit and implicit language. Journal of Gerontology, 1981, 36, 316-322.

Birren, J. E., Riegel, K. F., \& Robbin, J. S. Age differences in continuous word associations measured by speech recording. Journal of Gerontology, 1962, 17, 95-96.

Bower, G. H., Black, J. B., \& TURne R, T. J. Scripts in memory for text. Cognitive Psychology, 1979, 11, 177-220.

Bransfond, J. D., \& Johnson, M. K. Contextual prerequisites for understanding: Some investigations of comprehension and recall. Journal of Verbal Learning and Verbal Behavior, 1972, 11, 717-726.

Britton, B. K., \& Tesser, A. Effects of prior knowledge on use of cognitive capacity in three complex cognitive tasks. Journal of Verbal Learning and Verbal Behavior, 1982, 21, 421-436.

Burke, D. M., \& YEE, P. L. Semantic priming during sentence processing by young and older adults. Developmental Psychology, in press.

Charness, N. Aging and skilled problem solving. Journal of Experimental Psychology: General, 1981, 110, 21-38.

Clark, H. H., \& Haviland, S. E. Comprehension and the givennew contract. In R. O. Freedle (Ed.), Discourse production and comprehension. Norwood, N.J: Ablex, 1977.

Cohen, G. Language comprehension in old age. Cognitive $P_{s y-}$ chology, 1979, 11, 412-429.

Cohen, G. Inferential reasoning in old age. Cognition, 1981, 9, 59-72.

CraIK, F. I. M. Age differences in human memory. In J. E. Birren $\& \mathrm{~K}$. W. Schaie (Eds.), Handbook of the psychology of aging. New York: Van Nostrand Reinhold, 1977.

Craik, F. I. M., \& Simon, E. Age differences in memory: The roles of attention and depth of processing. In $\mathrm{L}$. W. Poon, J. L. Fozard, L. S. Cermak, D. Arenberg, \& L. W. Thompson (Eds.), New directions in memory and aging: Proceedings of the George Talland memorial conference. Hillsdale, N.J: Erlbaum, 1980.

Denney, N. W., \& Palmer, A. M. Adult age differences on traditional and practical problem-solving measures. Journal of Gerontology, 1981, 36, 323-328.
Dixon, R. A., Simon, E. W., Nowak, C. A., \& Hultsch, D. F. Text recall as a function of level of information, input modality, and delay interval. Journal of Gerontology, 1982, 37, 358-364.

Drachman, D. A., \& LeavitT, J. Memory impairment in the aged: Storage versus retrieval deficit. Journal of Experimental Psychology, 1972, 93, 302-308.

Eysenck, M. W. Age differences in incidental learning. Developmental Psychology, 1974, 10, 936-941.

Feier, C. D., \& Gerstman, L. J. Sentence comprehension abilities throughout the adult life span. Journal of Gerontology, $1980,35,722-728$.

Gordon, S. K., \& Clark, W. C. Application of signal detection theory to prose recall and recognition in elderly and young adults. Journal of Gerontology, 1974, 29, 64-72.

Graesser, A. C., Gordon, S. E., \& Sawyer, J. D. Recognition memory for typical and atypical actions in scripted activities: Tests of a script pointer + tag hypothesis. Journal of Verbal Learning and Verbal Behavior, 1979, 18, 319-332.

Graesser, A. C., Woll, S. B., Kowalski, D. J., \& Smith, D. A. Memory for typical and atypical actions in scripted activities. Journal of Experimental Psychology: Human Learning and Memory, 1980, 6, 503-515.

Grice, H. P. Logic and conversation. In P. Cole \& J. Morgan (Eds.), Syntax and semantics (Vol. 3): Speech acts. New York: Academic Press, 1975.

Harris, R. J., \& Monaco, G. E. Psychology of pragmatic implication: Information processing between the lines. Journal of Experimental Psychology: General, 1978, 107, 1-22.

Hasher, L., \& ZACKs, R. T. Automatic and effortful processes in memory. Journal of Experimental Psychology: General, 1979, 108, 356-388.

Hastie, R., \& Kumar, P. A. Person memory: Personality traits as organizing principles in memory for behaviors. Journal of Personality and Social Psychology, 1979, 37, 25-38.

HowARD, D. V. Category norms: A comparison of the Battig and Montague (1969) norms with the responses of adults between the ages of 20 and 80. Journal of Gerontology, 1980, 35, 225-231.

Light, L. L., Zelinski, E. M., \& Moone, M. Adult age differences in reasoning from new information. Journal of Experimental Psychology: Learning, Memory, and Cognition, 1982, 8, 435-447.

Lovelace, E. A., \& Cooley, S. Free associations of older adults to single words and conceptually related word triads. Journal of Gerontology, 1982, 37, 432-437.

Minsky, M. A framework for representing knowledge. In P. H. Winston (Ed.), The psychology of computer vision. New York: McGraw-Hill, 1975.

Perlumteren, M. What is memory aging the aging of? Developmental Psychology, 1978, 14, 330-345.

Perlmutte R, M. Age differences in the consistency of adults' associative responses. Experimental Aging Research, 1979, 5, 549-553.

Perlmutter, M., \& Mitchell, D. B. The appearance and disappearance of age differences in adult memory. In F. I. M. Craik \& S. Trehub (Eds.), Aging and cognitive processes. New York: Plenum, 1982.

Rabinowitz, J. C., Craik, F. I. M., \& Ackerman, B. P. A processing resource account of age differences in recall. Canadian Journal of Psychology, 1982, 36, 325-344.

RiEGel, K. F., \& Birren, J. E. Age differences in verbal associations. Journal of Genetic Psychology, 1966, 108, 153-170.

RIEGEL, K. F., \& RIEGEL, R. M. Changes in associative behavior during later years of life: A cross sectional analysis. Vita $\mathrm{Hu}$ mana, 1964, 7, 1-32.

Schate, K. W., \& Parham, I. A. Cohort-sequential analyses of adult intellectual development. Developmental Psychology, $1977,13,649-653$.

Schaie, K. W., \& Strother, C. R. A cross-sequential study of age changes in cognitive behavior. Psychological Bulletin, 1968, 70, 671-680. 
Schank, R. C., \& Abelson, R. P. Scripts, plans, goals, and understanding. Hillsdale, N.J: Erlbaum, 1977.

Schonfield, D., \& Stones, M. J. Remembering and aging. In J. F. Kihlstrom \& F. J. Evans (Eds.), Functional disorders of memory. Hillsdale, N.J: Erlbaum, 1979.

Shipley, W. C. A self-administering scale for measuring intellectual impairment and deterioration. Journal of Psychology, $1940,9,371-377$.

Simon, E. Depth and elaboration of processing in relation to age. Journal of Experimental Psychology: Human Learning and Memory, 1979, 5, 115-124.

Simon, E. W., Dixon, R. A., Nowak, C. A., \& Hultsch, D. F. Orienting task effects on text recall in adulthood. Journal of Gerontology, 1982, 37, 575-580.

Stones, M. J. Aging and semantic memory: Structural age differences. Experimental Aging Research, 1978, 4, 125-132.

TAUB, H. A. Comprehension and memory of prose materials by young and old adults. Experimental Aging Research, 1979, 5, 3-13.

TAUB, H. A., \& KLINE, G. E. Recall of prose as a function of age and input modality. Journal of Gerontology, 1978, 33, 725-730.

TiLl, R. E., \& Walsh, D. A. Encoding and retrieval factors in adult memory for implicational sentences. Journal of Verbal Learning and Verbal Behavior, 1980, 19, 1-16.

\section{NOTES}

1. Vocabulary scores for three young adults were unavailable.

2. Conclusions drawn from the recognition data do not depend on the choice of $\mathrm{d}^{\prime}$ here. Analysis of the data using $\mathrm{A}_{\mathrm{g}}$ led to rejection of the same null hypotheses at the same levels of significance.

3. Our results do differ from those of Graesser et al. (1980, Experiment 2) in some respects. Graesser et al. (1980) reported that the probability of making an intrusion correlated highly $(r=.67)$ with the probability of generating an item but not with rated typicality $(\mathrm{r}=.03)$, whereas the probability of making a false alarm correlated highly with rated typicality $(\mathrm{r}=.42)$ but not with generation probability $(r=.19)$. These correlations were computed only for the sample of typical actions in the Graesser et al. (1980) study (mean typicality values ranged from 4.4 to 6.0 ). When we computed these correlations for our sample of typical actions (mean typicality values ranged from 4.9 to 6.0 ), we found the same pattern of results for recall, with intrusions correlated .43 with generation probability and .01 with typicality. For recognition, we found nonsignificant correlations of false alarms with both typicality $(\mathrm{r}=-.17)$ and generation frequency $(\mathrm{r}=-.13)$. However, when we included all items, rather than only typical items, from the Jack story, the incidence of intrusions and false alarms was reliably correlated with generation frequency $(r=.49$ for intrusions and $r=.60$ for false alarms) and typicality $(r=.29$ for intrusions and $r=.57$ for false alarms). Graesser et al. (1980) argued that their results provide support for the view that retrieval processes are different in recall and in recognition. However, our failure to replicate Graesser et al.'s (1980) results when we included actions covering the full range of rated typicality suggests that this conclusion may not be warranted. Recall and recognition may involve different retrieval processes, but our data offer no support for this view.

(Manuscript received January 10,1983; revision accepted for publication May 3, 1983.) 